\title{
Angiostatic kinase inhibitors to sustain photodynamic angio-occlusion
}

\author{
Patrycja Nowak-Sliwinska ${ }^{a}{ }^{*}$, Andrea Weiss ${ }^{b}$, Judy R. van Beijnum ${ }^{\text {a }}$, Tse J. Wong ${ }^{a}$, \\ Jean-Pierre Ballini ${ }^{b}$, \#, Blaise Lovisa ${ }^{b}$, Hubert van den Bergh ${ }^{b}$, Arjan W. Griffioen ${ }^{a}$ \\ ${ }^{a}$ Angiogenesis Laboratory, Department of Medical Oncology, VU University Medical Center, Amsterdam, The Netherlands \\ ${ }^{b}$ Medical Photonics Group, Institute of Bioengineering, Swiss Federal Institute of Technology (EPFL), Lausanne, Switzerland
}

Received: June 10, 2011; Accepted: August 17, 2011

\begin{abstract}
Targeted angiostatic therapy receives major attention for the treatment of cancer and exudative age-related macular degeneration (AMD). Photodynamic therapy (PDT) has been used as an effective clinical approach for these diseases. As PDT can cause an angiogenic response in the treated tissue, combination of PDT with anti-angiogenic compounds should lead to improved therapy. This study was undertaken to test the clinically used small molecule kinase inhibitors Nexavar ${ }^{\circledR}$ (sorafenib), Tarceva ${ }^{\circledR}$ (erlotinib) and Sutent ${ }^{\circledR}$ (sunitinib) for this purpose, and to compare the results to the combination of Visudyne ${ }^{\circledR}$-PDT with Avastin ${ }^{\circledR}$ (bevacizumab) treatment. When topically applied to the chicken chorioallantoic membrane at embryo development day (EDD) 7, a clear inhibition of blood vessel development was observed, with sorafenib being most efficient. To investigate the combination with phototherapy, Visudyne ${ }^{\circledR}$-PDT was first applied on EDD11 to close all $<100 \mu \mathrm{m}$ vessels. Application of angiostatics after PDT resulted in a significant decrease in vessel regrowth in terms of reduced vessel density and number of branching points $/ \mathrm{mm}^{2}$. As the $50 \%$ effective dose (ED50) for all compounds was approximately 10 -fold lower, Sorafenib outperformed the other compounds. In vitro, all kinase inhibitors decreased the viability of human umbilical vein endothelial cells. Sunitinib convincingly inhibited the in vitro migration of endothelial cells. These results suggest the therapeutic potential of these compounds for application in combination with PDT in anti-cancer approaches, and possibly also in the treatment of other diseases where angiogenesis plays an important role.
\end{abstract}

Keywords: anti-angiogenic $\bullet$ bevacizumab $\bullet$ CAM model $\bullet$ Image $\bullet$ kinase inhibitor $\bullet$ photodynamic therapy

\section{Introduction}

Angiogenesis is a key step in physiological processes such as wound healing and the female reproductive cycle, as well as in pathologies such as cancer, rheumatoid arthritis and exudative AMD [1, 2]. The first successful large-scale treatment option for exudative AMD was verteporfin (Visudyne ${ }^{\circledR}$ ) PDT [3]. This approach is based on light-induced angio-occlusion after injection

\footnotetext{
\#This manuscript is dedicated to the memory of Dr. Jean-Pierre Ballini (1943-2010) who was a member of the Medical Photonics Group for many years and whose personality and work was invaluable to the laboratory and many of its endeavours.

${ }^{*}$ Correspondence to: Patrycja NOWAK-SLIWINSKA,

Urology Department, University Hospital (CHUV),

Lausanne, $\mathrm{CH}-1011$, Switzerland.

Tel.: +41-21-6935169

Fax: $+41-21-6935110$

E-mail: Patrycja.Nowak-Sliwinska@epfl.ch
}

of a photosensitizer. Several studies have shown that PDT can occlude experimental subfoveal choroidal neovascularization (CNV) with minimal damage to the surrounding retina [4]. Unfortunately, following verteporfin-PDT, a later angiogenic response can occur that limits the value of the treatment [5]. This has lead to renewed interest in understanding the mechanism of PDT and improving the basis of it $[6,7]$.

Angiogenesis occurs through an intricately regulated cascade of events involving, among other factors, the families of VEGFs, fibroblast growth factors (FGF), and also platelet-derived growth factor (PDGF) and epidermal growth factor (EGF) [8, 9]. Their receptors are linked to a tyrosine kinase domain that takes part in signalling pathways affecting endothelial cell (EC) proliferation, migration or differentiation ability [10], thus facilitating blood vessel regrowth. It is well-established that various pathologies are caused by overstimulation of tyrosine kinase activity and the intracellular signalling pathways that they activate. The activity does 
not have to be restricted to ECs, as PDGF signalling also appears to regulate the recruitment of support cells (smooth muscle cells/pericytes) to the vascular bed [11].

A number of kinase inhibitors are presently on the market and their application in clinical medicine is being elucidated [12]. Several kinase inhibitors are approved in cancer treatment: Gleevec ${ }^{\circledR} /$ imatinib (Novartis AG, Basel, Switzerland) for chronic myelogenous leukaemia and gastrointestinal stromal tumours [13], Tasigna ${ }^{\circledR} /$ nilotinib (Novartis) for chronic myelogenous leukaemia, including the patients resistant or intolerant to imatinib [14], Sutent ${ }^{\circledR} /$ sunitinib (Pfitzer Icl., New York, NY, USA) for renal cell carcinoma and gastrointestinal stromal tumours [15], Nexavar ${ }^{\circledR} /$ sorafenib (Bayer HealthCare and Onyx Pharmaceuticals, Inc.) for renal cell carcinoma and hepatocellular carcinoma [16], and Iressa ${ }^{\circledR} /$ gefitinib (Astra/Zeneca, London, UK) for non-small cell lung cancer [17]. More than 20 other kinase inhibitors are currently in clinical trials, also for other diseases than cancer [18]. These inhibitors target either mainly single receptors, for example erlotinib, which binds EGFR [19], or are broad spectrum inhibitors, for example sorafenib, which targets B-Raf/VEGFR-2/NEGFR-3/PDGFR-b/FLT-3/c-KIT/RAF [20] or sunitinib inhibiting VEGFR/PDGFR-b/c-KIT/FLT-3 [21].

As inhibition of angiogenesis has been recognized as a promising treatment strategy of angiogenic diseases, an increasing number of anti-angiogenic compounds are being developed for use in either mono- or combination therapies [22]. Thus, in this study we investigated the efficacy of selected kinase inhibitors, both on the morphological and molecular level, following angio-occlusive PDT.

An attractive model for studying angiogenesis after PDT is the chorioallantoic membrane (CAM) of the chicken embryo. Study of photodynamic angio-occlusion and reperfusion, as well as neoformation of vessels can be performed in situ and in real time [23-26]. This in vivo pre-clinical model has the advantage of having a thin, planar vascular network, which is well-accessible to PDT and PDT in combination with adjuvants, added either topically or intravenously (i.v.) [27].

In this study we adopted a previously described automated quantification method [25] to assess the vessel regrowth rate in the PDT-treated area of the CAM, using high-quality fluorescence angiography of the revascularization in the PDT-treated area. The method was used to show the prolonged angio-occlusive effect after PDT, when topically applying the angiogenesis inhibitors.

\section{Materials and methods}

\section{Materials and chemicals}

Avastin ${ }^{\circledR}$ was obtained from Genentech (San Francisco, CA, USA) and Sutent ${ }^{\circledR}$ from Pfizer Inc. (New York, NY, USA). Nexavar ${ }^{\circledR}$ and Tarceva ${ }^{\circledR}$ were obtained from LC Laboratories (Woburn, MA, USA). Visudyne ${ }^{\circledR}$ (the liposomal formulation of verteporfin) was generously provided by Novartis Ophthalmics (Hettlingen, Switzerland). Fluorescein isothiocyanate dextran (FITC-dextran, 20 kD) was purchased from Sigma-Aldrich (Buchs,
Switzerland). The $0.9 \% \mathrm{NaCl}$ solution, which was used as a solvent for the kinase inhibitors, or by itself as the control, is a product of Bichsel $A G$ (Interlaken, Switzerland). Embryos were obtained from Animalco $A G$ (Staufen, Switzerland). India ink was purchased at Pelikan (Witzikon, Switzerland) and filtered through a sterile cellulose acetate membrane $(0.2$ $\mu \mathrm{m}$ pores; Renner GmbH, Darmstadt, Germany). The injections in the CAM were performed with Microliter ${ }^{\mathrm{TM}}$ syringes equipped with 33-gauge metal Hub (N) needles, both from Hamilton (Reno, NV, USA).

\section{Developmental CAM and quantification of the angiogenic response}

In this assay, the anti-angiogenic efficacy of the compounds was tested in the physiologically developing CAM model between EDD7 and EDD9, as previously described in detail [25]. Briefly, compounds were applied topically twice (each time $20 \mu \mathrm{l}$ ) on EDD7 and EDD8. The concentrations ranged from 0.1 to $300 \mu \mathrm{M}$ (this corresponds to $0.001-2.6 \mu \mathrm{g} / \mathrm{embryo}$ for erlotinib and $0.0013-3.9 \mu \mathrm{g} /$ embryo for sorafenib), from 2 to $1000 \mu \mathrm{M}$ (0.0213-10.65 $\mu \mathrm{g} / \mathrm{embryo})$ for sunitinib, and from 0.2 to $80 \mu \mathrm{M}$ for bevacizumab (0.6-238 $\mu \mathrm{g} / \mathrm{embry})$ ). The control eggs received $0.9 \% \mathrm{NaCl}$ twice (each time $20 \mu \mathrm{l}$ ). On EDD9, the CAMs were visualized in ovo by means of FITC-dextran $(25 \mathrm{mg} / \mathrm{ml}, 20 \mu \mathrm{l})$ epi-fluorescence angiography and subsequently analysed by the image-processing quantification method described later. At least five eggs were tested per condition.

\section{Visudyne ${ }^{\circledR}$-PDT in combination with topically administrated tyrosine kinase inhibitors}

We also combined Visudyne ${ }^{\circledR}$-PDT with the subsequent topical administration of the following angiogenesis inhibitors: bevacizumab (2-20 $\mu \mathrm{M}$ corresponding to $6-60 \mu \mathrm{g} / \mathrm{embry})$, sunitinib $(2-20 \mu \mathrm{M}$ corresponding to 0.02-0.2 $\mu \mathrm{g} / \mathrm{embryo})$, sorafenib (2-20 $\mu \mathrm{M}$ corresponding to $0.026-0.26$ $\mu \mathrm{g} / \mathrm{embry0}$ ), erlotinib (1-20 $\mu \mathrm{M}$ corresponding to $0.01-0.2 \mu \mathrm{g} / \mathrm{embryo}$ ). These anti-angiogenic compounds were deposited topically to the surface of the CAM in the form of liquid drops of $20 \mu \mathrm{l}$ within a polyethylene ring (diameter $5 \mathrm{~mm}$; wall thickness $0.5 \mathrm{~mm}, 1 \mathrm{~mm}$ height). All tested compounds were applied twice, immediately after PDT $(20 \mu \mathrm{l})$, and $24 \mathrm{hrs}$ after PDT $(20 \mu \mathrm{l})$. As mentioned before, fluorescence angiographies were taken 24 and 48 hrs after either PDT or combined treatment.

\section{Microscopy and image acquisition}

Microscopic observation of CAM vasculature, as well as the light irradiation during PDT, were performed with an epi-fluorescence Eclipse $600 \mathrm{FN}$ microscope equipped with a Plan Apo $4 \times / 0.2$, working distance of $20 \mathrm{~mm}$ or Plan Fluor $10 \times / 0.3$, working distance of $16 \mathrm{~mm}$ objectives (Nikon, Tokyo, Japan). Illumination was provided by a $100 \mathrm{~W}$ high pressure $\mathrm{Hg}$-arc lamp, as described before [23]. Light doses were adjusted with neutral density filters and measured with a calibrated Field-Master GS power meter (Coherent, Santa Clara, CA, USA). For exciting and detecting Visudyne ${ }^{\circledR}$, the microscope was equipped with a BV-2A filter set $\left(\lambda_{e x}=420 \pm 20 \mathrm{~nm}\right.$, $\lambda_{\text {em }}>470 \mathrm{~nm}$; Nikon). For detecting FITC, light was filtered for excitation at $470 \pm 20 \mathrm{~nm}$ and a long-pass emission filter was used for detection of the fluorescence ( $\lambda>520 \mathrm{~nm}$; Nikon). Fluorescence images were acquired with an F-view II 12-bit monochrome Peltier-cooled digital charge-coupled 
device camera driven with analySIS DOCU software from Soft Imaging System (Munster, Germany) [26, 28].

\section{The in ovo CAM model for Visudyne ${ }^{\circledR}$-PDT}

Fertilized chicken eggs were labelled and transferred into a hatching incubator with a relative air humidity of $65 \%$ and a temperature of $37^{\circ} \mathrm{C}$, as described previously $[23,25]$. On EDD3, a hole of approximately $3 \mathrm{~mm}$ in diameter was opened in the eggshell and covered with a laboratory wrapping film, Parafilm ${ }^{\circledR}$ (Pechiney, Menasha, WI, USA) to prevent dehydration and possible infections.

On EDD11, the egg opening was extended to $3 \mathrm{~cm}$ in diameter, and Visudyne ${ }^{\circledR}$ was i.v. administered into the main vessel of the CAM. Eggs were placed under the epi-fluorescence microscope and $1 \mathrm{~min}$. after injection, the CAM was irradiated with a light dose of $20 \mathrm{~J} / \mathrm{cm}^{2}\left(\lambda_{\text {ex }}=420 \pm 20 \mathrm{~nm}\right)$ and irradiance $50 \mathrm{~mW} / \mathrm{cm}^{2}$. The irradiation area was limited to $0.02 \mathrm{~cm}^{2}$ by an optical diaphragm within the area defined by the polyethylene ring used for the topical drug administration. Visudyne ${ }^{\circledR}$-based fluorescence angiographies were taken before and right after irradiation $\left(\lambda_{\mathrm{ex}}=420 \pm 20 \mathrm{~nm}\right)$. The PDT conditions we used led to angio-occlusion efficiency for the CAM vessels similar to those observed clinically after Visudyne ${ }^{\circledR}$-PDT in the human eye, that is similar diameters of vessels were closed in the CAM as in the human CNV. Photodynamic therapy induced closure of the CAM-vasculature is defined as optimal when vessels with a diameter $\leq 70 \mu \mathrm{m}$ are closed, while leaving the larger vessels open [23, 29]. Fluorescein isothiocyanate based fluorescence angiographies were taken 24 and 48 hrs after PDT after i.v. injection of $20 \mu \mathrm{l}$ of FITC-dextran $(20 \mathrm{kD}, 25 \mathrm{mg} / \mathrm{ml})$. Prior to the fluorescence angiography, an India ink injection in the extra-embryonic cavity was performed to block optical interference from the autofluorescence originating in the embryo below the CAM. For these angiographies, we used light from a filtered $\mathrm{Hg}$-arc lamp for excitation ( $\lambda_{\mathrm{ex}}=470 \pm 20 \mathrm{~nm}$ ), and a longpass emission filter $\left(\lambda_{e m}>520 \mathrm{~nm}\right)$, as described later [23].

\section{Quantitative image analysis}

Fluorescence angiographies of the CAM at EDD9 were analysed using a macro written for ImageJ, as previously described [25]. Briefly, images were quantified using a two-step processing algorithm. The first phase extracted the large vessels with a threshold-based segmentation. The second phase extracted the small vessels and capillaries using a rolling-ball background filter. The results from both phases were then combined and skeletonized to describe the vessel connectivity.

In this study, the above-mentioned macro was adapted to quantify the PDT-treated areas of the CAM (EDD13). Four concentric regions-of-interests were defined to be able to quantify the treatment efficacy from the border to the center (Fig. 3B). Area 4, the most external zone is located on the edge of the PDT-treated zone and gets revascularized first. Area 1 is the most inner location within the PDT-treated area, and gets revascularized last. To monitor the vascular network and the regrowth of the vasculature within the PDT-treated area, three selected descriptors were calculated by our software. The descriptors are defined as follows: Descriptor 1 is the number of branching points $/ \mathrm{mm}^{2}$. Descriptor 2 is a delimiter of vascular mesh size, expressed as the $3^{\text {rd }}$ quartile of the shortest distance from any pixel to the nearest segment and given in micrometers. This descriptor gives information on the capacity of oxygenation of the tissue areas, allowing the generation of a hypoxia map (Fig. 3). Descriptor 3 represents the vessel density. This parameter is based on the number of black pixels in the skeletonized image, scaled per surface of each analysed ring and expressed as a percentage of this surface.

\section{RNA isolation, cDNA synthesis and primers}

Total RNA was isolated from CAM sections treated with mono- or combination therapies using the TRIzol Reagent (Invitrogen, Carlsbad, CA, USA) according to the supplier's protocol. RNA quality and quantity were checked by spectrophotometry (NanoDrop; Thermo Scientific, Breda, The Netherlands), and $1000 \mathrm{ng}$ RNA was used as input for first-strand cDNA synthesis using an iScript cDNA synthesis kit (Bio-Rad Laboratories, Veenendaal, The Netherlands) according to the manufacturers' instructions. Reactants were diluted to $50 \mu \mathrm{l}$ and stored at $-20^{\circ} \mathrm{C}$ until use.

The primers were specific for chicken (Gallus gallus) sequences and selected based on the following requirements: (i) GC content of approximately $55 \%$, (ii) primer melting temperature of approximately $60^{\circ} \mathrm{C}$, (iii) preferably no $\mathrm{G}$ at the 5 ' end, (iv) avoid runs of more than three identical nucleotides and (v) amplicon length of approximately 100 nucleotides. Specificity and cross-reactivity were checked with the Basic Local Alignment Search Tool (BLAST; http:// www.ncbi.nlm.nih.gov/BLAST). Primers were synthesized by Eurogentec (Liège, Belgium) and targeted against $\beta$-actin (Actin- $\beta$ ), cyclophilin-A (Cyclo-A), vascular endothelial growth factor $A$ (VEGFA), vascular endothelial growth factor receptors 1 and 2 (VEGFR1, -2), neuropilin-2 (NRP2), basic fibroblast growth factor (bFGF), integrin $\beta 3$ (ITGB3) and galectin 1 (GAL1).

\section{Quantitative real-time RT-PCR}

Quantitative real-time RT-PCR (qRT-PCR) was performed in $25 \mu \mathrm{l}$ reactions, containing $1 \times$ iQ SYBR Green supermix (Bio-Rad Laboratories), 200 $\mathrm{nM}$ forward and reverse primer (Fig. $7 \mathrm{C}$ ) and $1.5 \mu \mathrm{l}$ CDNA. Reactions were run on a CFX96 thermal cycler (Bio-Rad Laboratories), and consisted of an initial denaturation for $10 \mathrm{~min}$. at $95^{\circ} \mathrm{C}$ followed by 50 cycles of 15 seconds at $95^{\circ} \mathrm{C}$ and $1 \mathrm{~min}$. at $60^{\circ} \mathrm{C}$. Following each run, melting curves were generated to verify specific product formation. Data were analysed using CFX Manager software (Bio-Rad Laboratories) and the expression of each target gene was quantified relative to the expression of the reference genes (Actin- $\beta$ and Cyclophilin-A) [30].

\section{Cells and reagents}

Human umbilical vein ECs (HUVEC) were isolated from fresh human umbilical cord veins and maintained in RPMI-1640 (Invitrogen) supplemented with 10\% human serum, 10\% FCS, 1\% glutamin (Invitrogen), $100 \mathrm{IU} / \mathrm{ml}$ penicillin (Sigma-Aldrich, St. Louis, MO, USA), and $100 \mu \mathrm{g} / \mathrm{ml}$ streptomycin (Sigma-Aldrich) as previously described [31]. Cells were cultured in a highly humidified atmosphere with $5 \% \mathrm{CO}_{2}$ at $37^{\circ} \mathrm{C}$.

\section{Proliferation assay}

Endothelial cells $\left(5 \times 10^{3}\right.$ cells/well) were seeded in gelatin-coated 96 -well cell culture plates as described previously [32]. Briefly, 24 hrs after seeding, culture medium with or without compounds was added and cells were grown for a further $72 \mathrm{hrs}$. Cell viability was assessed using the CellTiterGlo ${ }^{\circledR}$ Luminescent Cell Viability Assay (Promega, Madison, WI, USA). 


\section{Wound assay (migration)}

The migration capability of ECs was measured using the wound assay [33]. In brief, HUVEC were grown to confluence in gelatin-coated wells and 'scratch wounds' (with an approximate width of $350 \mu \mathrm{m}$ ) were made in the monolayer by removing cells with a sterile scratch tool (Peira Scientific Instruments, Beerse, Belgium). Cells were washed with PBS and the medium was replaced by fresh medium containing $10 \mathrm{ng} / \mathrm{ml}$ bFGF (Tebu-Bio, Heerhugowaard, The Netherlands) and incubated with or without tyrosine kinase inhibitors. Automated image acquisition was performed with a Leica DMI3000B microscope in combination with UGR grabbing software (DCI Labs, Peira Scientific Instruments). Bright field images taken at $5 \times$ magnification were processed for computational analysis of scratch sizes using UGR Scratch Assay 6.2 software (DCI Labs, Peira Scientific Instruments).

\section{Statistical analysis}

Values are given as mean values \pm S.E.M. Data are represented as averages of independent experiments, performed in duplicate or triplicate. Statistical analyses were done using the Student's $t$-test. $P$-values $<0.05$ were considered statistically significant.

\section{Results}

\section{Inhibition of developmental angiogenesis in the CAM}

We tested the angiostatic activity of the kinase inhibitors: erlotinib, sorafenib and sunitinib in vivo in the CAM assay, and compared the results with those obtained after treatment with bevacizumab (Avastin $)^{Q}$. Dose ranges of compounds were administered topically on the CAM on EDD7, when vascularization of the membrane starts to become exponential. Treatment was repeated on EDD8 and the vascularization of the CAM was analysed and quantified on EDD9. Treatment with the compounds at low concentrations resulted in the presence of small avascular zones in the capillary bed along the larger blood vessels (Fig. 1). At higher concentrations, as of $30-50 \mu \mathrm{M}$ for TKIs and $10 \mu \mathrm{M}$ for bevacizumab, loss of vascular hierarchy was observed, as well as complete absence of vasculature within large areas of the CAM (Fig. 1C, arrows indicate the avascular zones, appearing as black areas in the fluorescence angiographies). For all inhibitors tested we observed a dose-dependent anti-angiogenic activity. As compared to the untreated control, statistically significant differences for both reported descriptors were observed as of $100 \mu \mathrm{M}$ (erlotinib $P=$ 0.045 , sorafenib $P=0.028$, sunitinib $P=0.048$ ). Digital analysis of the images revealed a slightly better activity for sorafenib over the other kinase inhibitors (versus sunitinib shown in Fig. 1 at $300 \mu \mathrm{M} ; P=0.044$ for branching points per $\mathrm{mm}^{2}$ and $P=0.027$ for mean mesh size). Assessment of the number of branching points per $\mathrm{mm}^{2}$ (Fig. 1A) and the relative mesh size (Fig. 1B) showed for sorafenib an $\mathrm{ED}_{50}$ of $100 \mu \mathrm{M}$, while this was approximately $300 \mu \mathrm{M}$ for erlotinib and sunitinib. The $\mathrm{ED}_{50}$ of bevacizumab in this assessment was $80 \mu \mathrm{M}$. Representative fluorescence angiographies of dose-dependent morphological changes are shown in Figure $1 \mathrm{C}$ for sunitinib (upper row) and bevacizumab (lower row).

\section{Morphological and molecular response in the CAM after PDT}

The CAM model is often used as a model for PDT-induced angioocclusion $[23,34]$. We observed that Visudyne ${ }^{\circledR}$-PDT, applied at EDD11, induced an efficient closure of the vasculature (Fig. $2 \mathrm{~A}$ and $\mathrm{B}$ ), as well as an angiogenic response in the treated tissue, compromising the angio-occlusive effect of therapy. Vascular regrowth in the CAM is visible $24 \mathrm{hrs}$ after PDT (Fig. 2B) and is completed $48 \mathrm{hrs}$ after PDT (Fig. 2C). The morphology of the neovessels was observed to be completely different from the normal vasculature and larger vascular meshes were observed, as compared to the non-PDT treated areas of the CAM. To investigate and prove the induction and regulation of angiogenesis after PDT, we determined gene expression before and $24 \mathrm{hrs}$ after PDT (EDD12) of a set of endothelial angiogenesis markers, such as galectin-1 (GAL1) and integrin $\beta$-3 (ITGB3), and the angiogenesis related genes for neuropillin-2 (NRP2), VEGFR1, -2, VEGF and bFGF. Expression was compared to the reference genes Actin- $\beta$ and Cyclophilin-A. We noticed a PDT-mediated induction of the endothelial angiogenesis markers galectin- 1 and integrin- $\beta 3$, as well as of neuropillin and the VEGF receptors.

\section{Post-PDT vascular regrowth inhibition by angiostatic kinase inhibitors}

To maintain and prolong the PDT-induced vaso-occlusive effect, we tested a series of clinically used angiostatic kinase inhibitors. Topical administration of $20 \mu \mathrm{l}$ of $20 \mu \mathrm{M}$ of sunitinib, sorafenib or erlotinib immediately after PDT, resulted in a statistically significant $(P<0.05)$ prolongation of PDT-induced vascular occlusion (seen as black zones on the angiographies). Figure $3 \mathrm{~A}$ shows representative angiographies taken 48 hrs after PDT without (left image) or in combination with topical administration of compounds. Results are compared with combination treatment with bevacizumab (right image). Interestingly, while regrown vasculature in the PDT treated area shows different morphology as compared to the original vascular bed, the morphology of the regrown vasculature in the presence of different compounds varied as well. Topical administration of sunitinib prevented the vascular regrowth in the central part of the PDT-treated area. Topical administration of sorafenib turned out to completely inhibit angiogenic response within PDT-treated zone. However, the morphology of the vasculature outside the PDT-treated was slightly affected as well. Both larger vessels and capillary bed remained occluded 48 hrs after treatment. Administration of the same concentration of erlotinib revealed a 
A

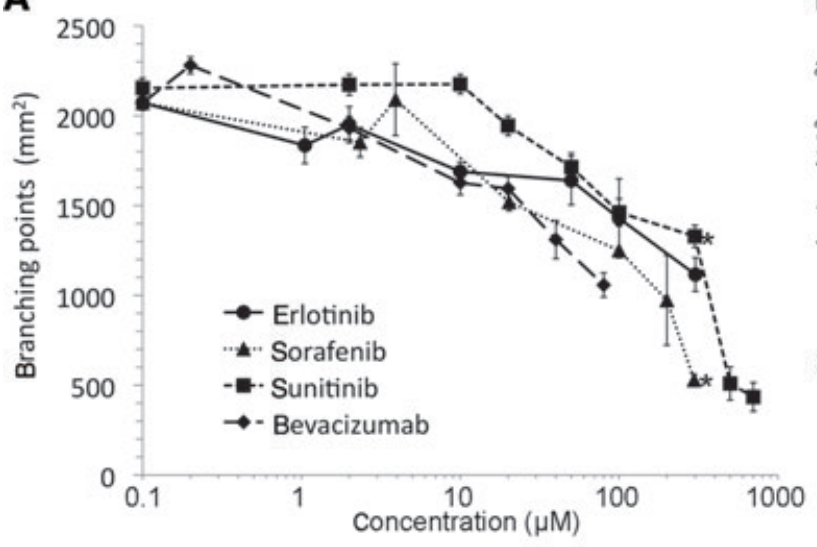

B

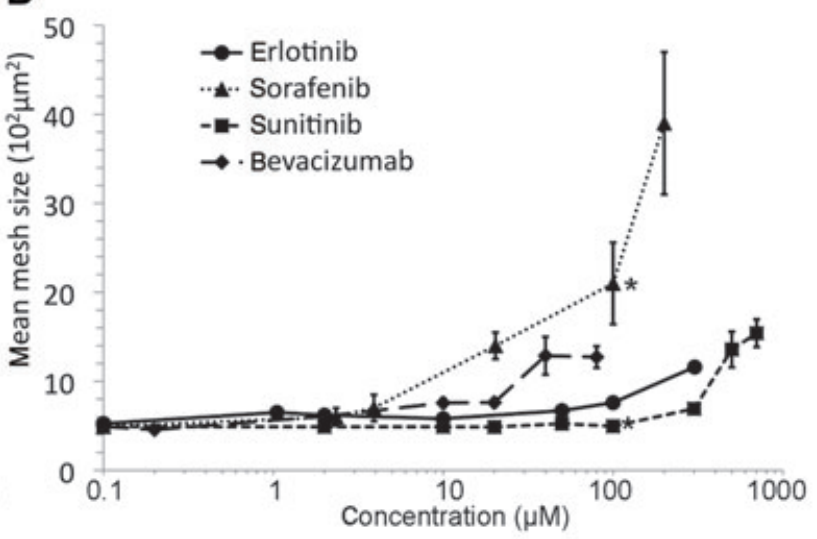

C
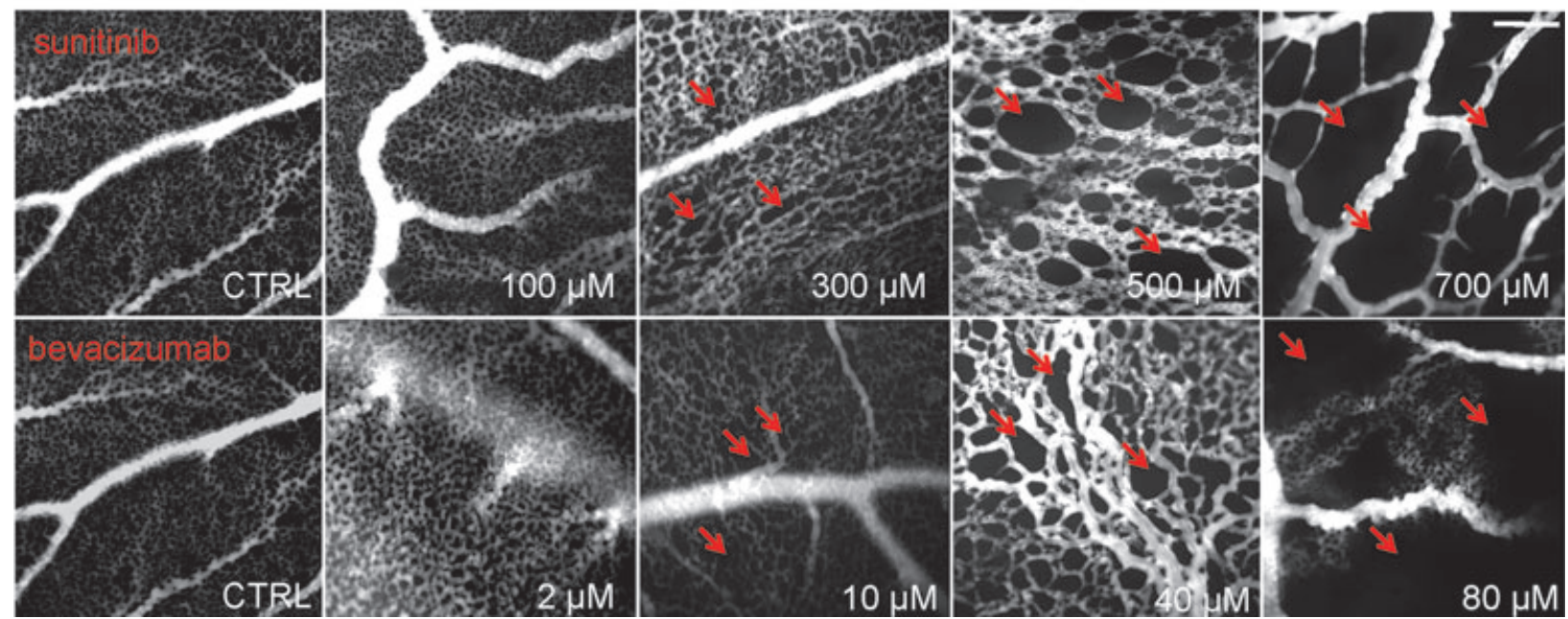

Fig. 1 Inhibition of developmental angiogenesis in the CAM by kinase inhibitors and bevacizumab. (A, B) Digital analysis of the vasculature in the CAM after treatment with kinase inhibitors or bevacizumab. (A) Inhibition of angiogenesis represented as inhibition of relative number of branching points $/ \mathrm{mm}^{2}$ as a function of drug concentration. Asterisks represent the statistical significance of the higher activity of sorafenib over sunitinib at $100 \mu \mathrm{M}$ of the compounds. (B) Inhibition of angiogenesis represented as an increase of mean vascular mesh size as a function of drug concentration. (C) Angiographic images of the developmental CAM treated with indicated concentrations (expressed in $\mu \mathrm{M}$ ) of sunitinib (upper row) and bevacizumab (lower row). The vasculature is visualized by FITC-dextran fluorescence angiography $(25 \mathrm{mg} / \mathrm{kg}, 20 \mathrm{kD}, \lambda$ ex $=470 \mathrm{~nm}, \lambda$ em $>520 \mathrm{~nm})$ on EDD9. The bar in the right image of the upper row represents $200 \mu \mathrm{m}$ and is valid for all images. Arrows indicate the avascular zones in the CAM, visible as a black areas in angiographies. Error bars represent standard error of the mean.

similar efficacy as sunitinib, however more sprouts were observed at the edge of PDT-treated area. Moreover, larger vessels seemed to be less sensitive to the treatment and were reperfused at $48 \mathrm{hrs}$. Bevacizumab inhibited angiogenesis, but to a lesser extend than the kinase inhibitors. For digital imaging and analysis of the vasculature in the PDT-treated area, we developed a quantitative image processing method to process the fluorescence angiographies. A schematic diagram of the method is shown in Figure 3B. The method allows independent analysis of circular regions of the PDT area, using three different descriptors, that is number of branching points $/ \mathrm{mm}^{2}$, relative vascular mesh size and vessel density. We found that all com- pounds inhibited vascular regrowth dose dependently, as assessed by all three descriptors. The most significant differences between the compounds were obvious in the inner most zones of the PDTtreated area (zones 1 and 2) and quantification of it is shown for zone 1 for two concentrations of the drugs (2 and $20 \mu \mathrm{M}$; Fig. 3C). The most distinguished results were visible for $20 \mu \mathrm{M}$, revealing superior activity for sorafenib ( $P<0.025$ versus sunitinib) over the other tested agents. All results obtained for combination therapy were statistically significant as compared to PDT alone. Moreover, we observed a statistically significant difference for applied concentrations of sorafenib after PDT (see asterisks in Fig. 3C) for 
Before Visudyne ${ }^{\circledR}$-PDT
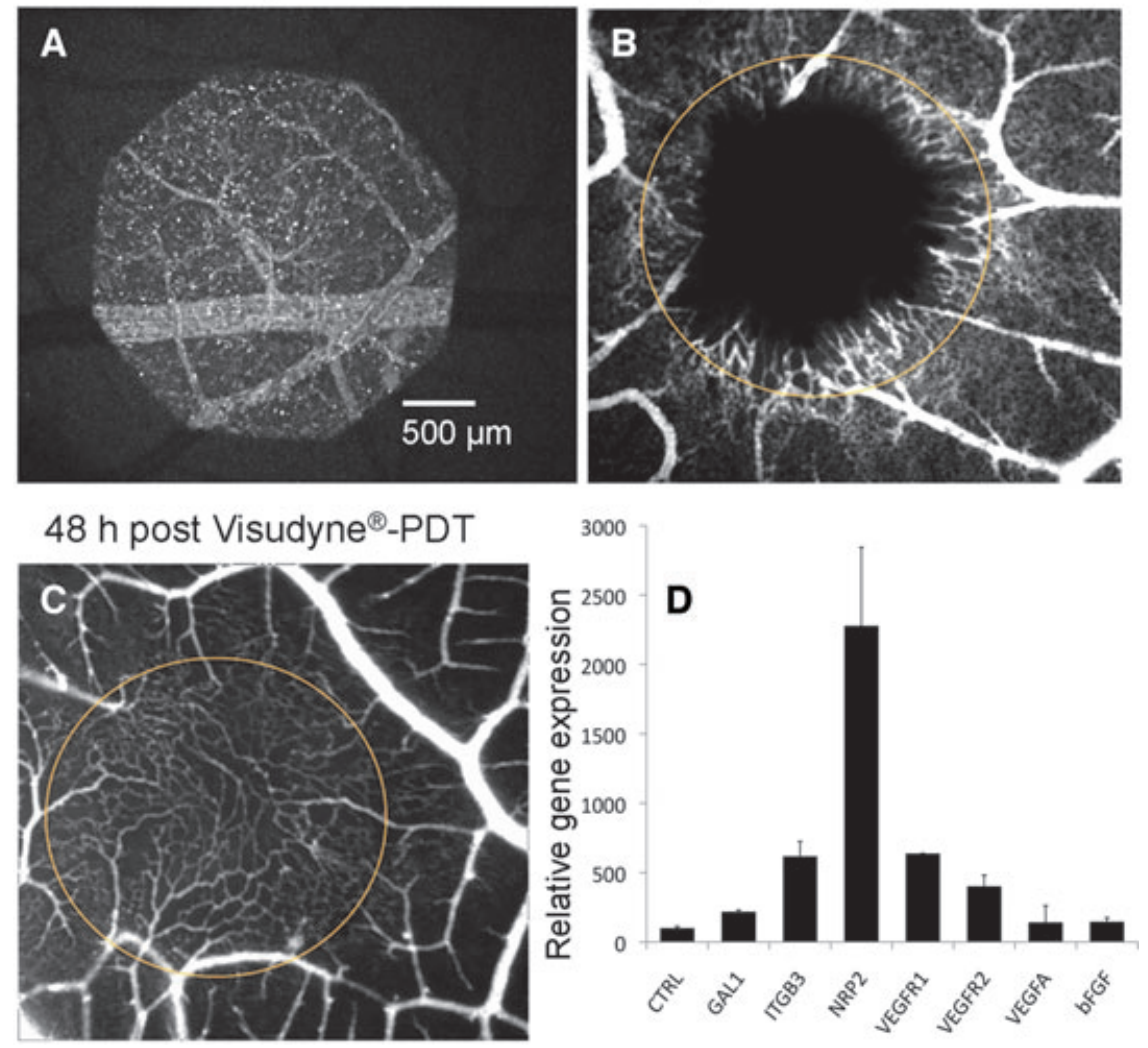

24 h post Visudyne ${ }^{\circledR}$-PDT

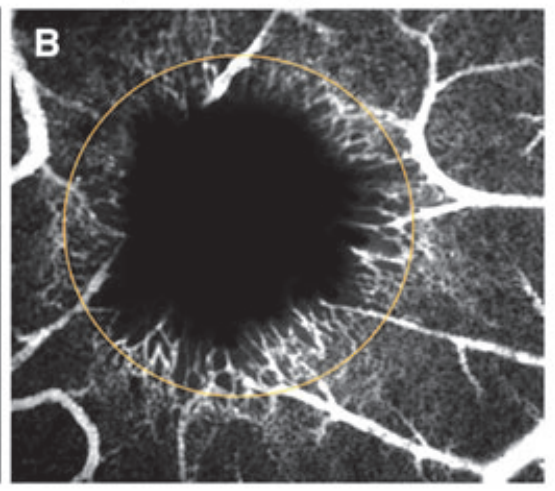

Fig. 2 Impact of PDT on morphological and molecular level of the mature CAM 24 hrs after treatment. Representative angiography images of the CAM before (A), 24 hrs (B) and 48 hrs after PDT (C). Vasculature in picture $A$ is visualized by Visudyne ${ }^{\circledR}$ fluorescence angiography $(0.20 \mathrm{mg} / \mathrm{kg}$ embryo weight, $\lambda_{e x}=420 \mathrm{~nm}, \lambda_{e m}>470 \mathrm{~nm}$ ). Pictures $B$ and $C$ are visualized by FITCdextran fluorescence angiography (25 $\mathrm{mg} / \mathrm{kg}, 20 \mathrm{kD}, \lambda_{\mathrm{ex}}=470 \mathrm{~nm}, \lambda_{\mathrm{em}}>520$ $n m)$. PDT was performed at a light dose of $20 \mathrm{~J} / \mathrm{cm}^{2}$ and an irradiance of $50 \mathrm{~mW} / \mathrm{cm}^{2}$. Black background colour is obtained by India ink injection $(30 \mu \mathrm{l})$ into the extraembryonic cavity right under the treated CAM area. Circles indicate the PDT-treated area. The scale bar in A applies for all panels. (D) Real-time quantitative RT-PCR molecular profiling of the CAM $24 \mathrm{hrs}$ after PDT. Relative transcript expression in the CAM versus the reference genes Actin- $\beta$ and Cyclophilin-A for the following treatment groups: control (CTRL), galectin-1 (GAL1), integrin $\beta 3$-subunit (ITGB3), neuropillin-2 (NRP2), VEGFR-1, -2, VEGF and bFGF. Error bars represent standard error of the mean. branching points $\left(\mathrm{mm}^{2}\right)$ with $P=0.032$, relative vascular mesh size ( $\mu \mathrm{m})$ with $P=0.027$, and vessel density with $P=0.026$.

The vascular regrowth was also monitored by quantitative real-time PCR analysis of the expression of VEGFR-1 and -2, being vascular angiogenesis markers. Expression of both VEGF receptors was up-regulated by PDT. The kinase inhibitors also induced their expression and co-treatment with kinase inhibitors did not change that (Fig. 4).

\section{TKIs, but not bevacizumab, inhibit endothelial cell growth and migration in vitro}

To compare the above results with the exclusive activity of the compounds on endothelial cells, we tested the compounds for their ability to inhibit human endothelial cell growth and migration. All kinase inhibitors decreased the viability of HUVEC with ED50 values of $2 \mu \mathrm{M}$ (erlotinib), $3 \mu \mathrm{M}$ (sunitinib) and $50 \mu \mathrm{M}$ (sorafenib). As expected, bevacizumab did not inhibit endothelial cell growth at any of the concentrations applied (Fig. 5A).

As the second indication of the compound's activities on EC function, migration assays were performed with HUVEC. As shown in Figure 5B, only sunitinib dose-dependently diminished the motility of EC at concentrations upwards from $1 \mu \mathrm{M}$, with an $\mathrm{ED}_{50}$-value of approximately $3 \mu \mathrm{M}$. Figure $5 \mathrm{C}$ shows examples of the wounds in the confluent HUVEC layer before (left image) and after 8 hrs of incubation with (1 or $30 \mu \mathrm{M})$ or without sunitinib.

\section{Discussion}

This study describes that PDT-induced vascular occlusion in the CAM can be prolonged by topical co-treatment with clinically used angiostatic small molecule kinase inhibitors. This maybe a relevant observation in the field of ocular pathology, as treatment of AMD is nowadays performed by regular intravitreal injection of ranibizumab (Lucentis ${ }^{\circledR}$ ) [35]. The CAM model has been shown to be a useful model to study retinal vasculature [23, 36]. It was previously observed that PDT can induce significant angioocclusion of the exposed CAM vasculature in vessels with a diameter of $<100 \mu \mathrm{m}$. This process coincided with induction of an angiogenic response resulting in complete revascularization of the angio-occluded area over a period of $48 \mathrm{hrs}$ [23]. We have previously demonstrated that angiostatic treatment of the CAM with 

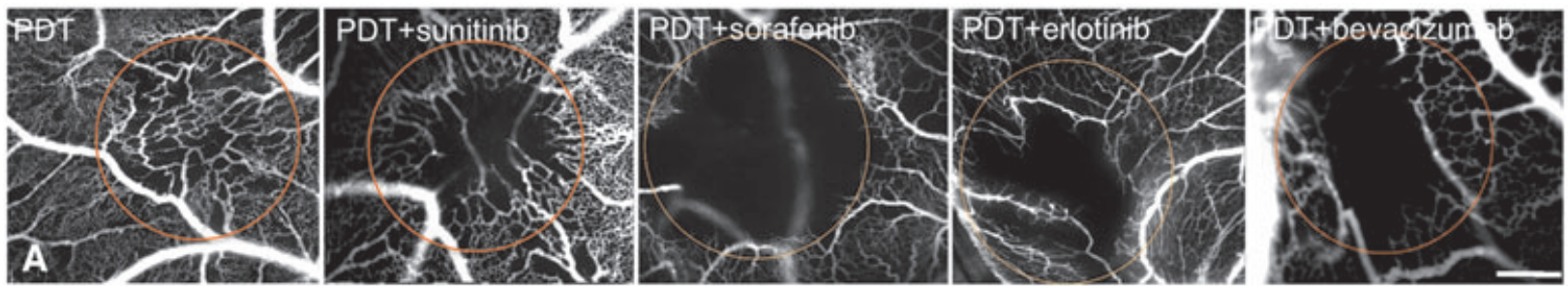

\section{Fluorescence angiogram}

\section{Skeleton}

\section{Potential oxygenation map}
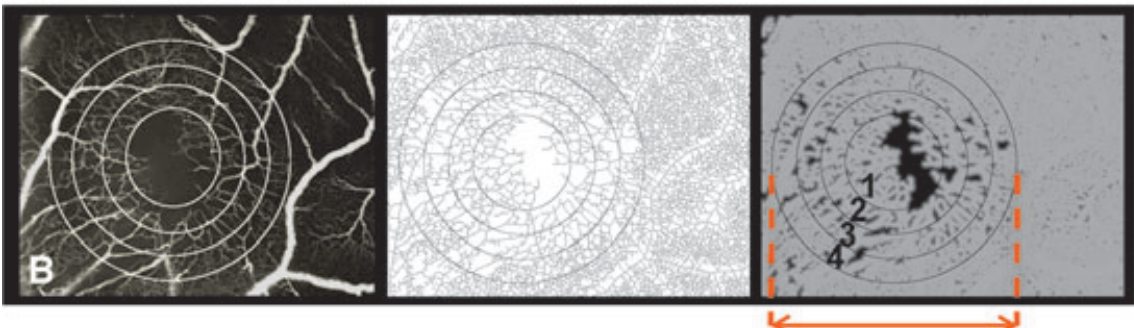

PDT-treated area containing 4 concentric circles
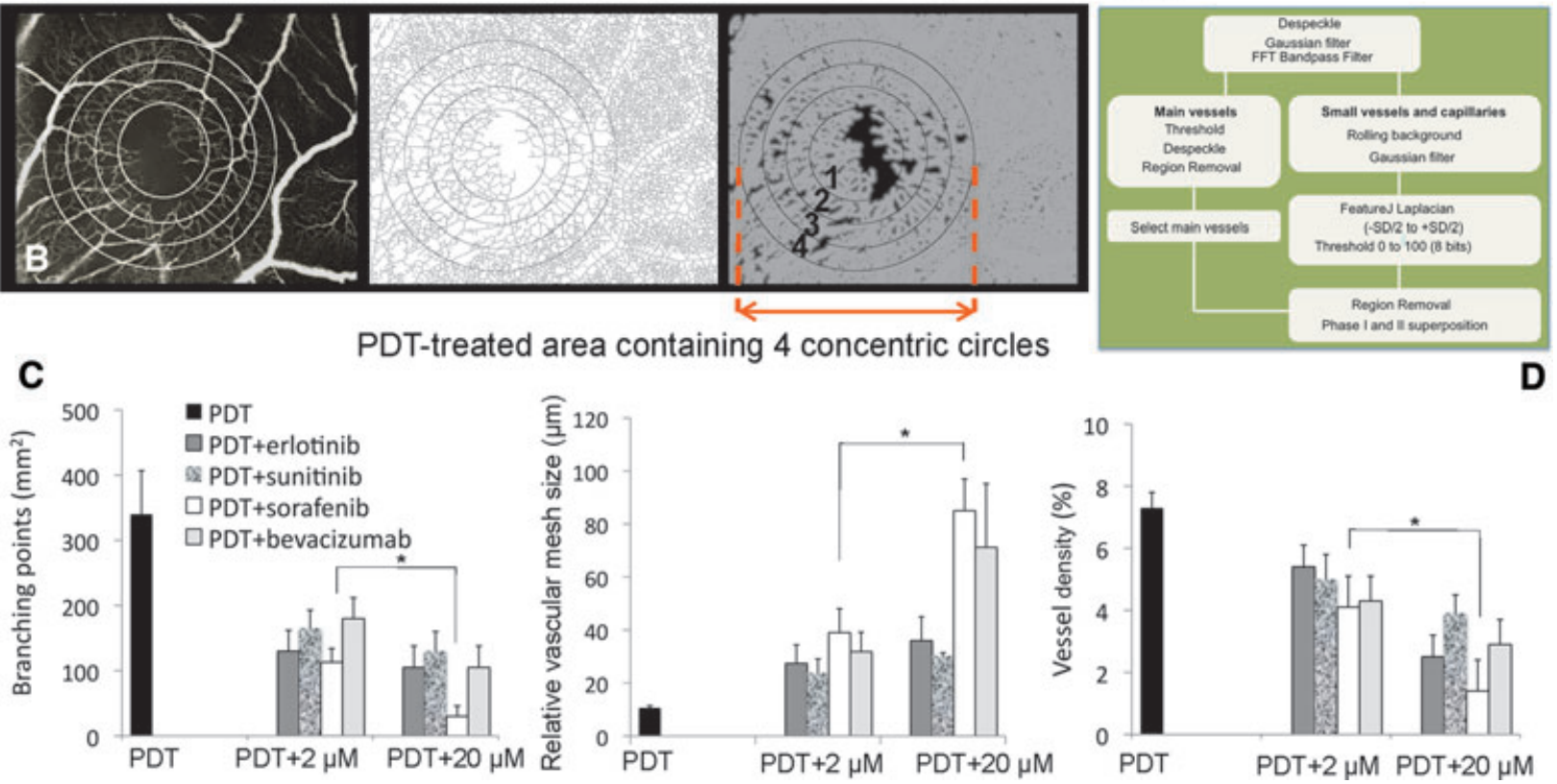

Fig. 3 Sustained photodynamic vaso-occlusion by angiogenesis inhibitors. (A) Typical fluorescence angiographies taken 48 hrs after Visudyne ${ }^{\circledR}$-PDT $\left(0.20 \mathrm{mg} / \mathrm{kg}\right.$ embryo weight, $\lambda_{\mathrm{ex}}=420 \mathrm{~nm}, \lambda_{\mathrm{em}}>470 \mathrm{~nm}$; light dose of $20 \mathrm{~J} / \mathrm{cm}^{2}$ and an irradiance of $50 \mathrm{~mW} / \mathrm{cm}^{2}$, drug-light interval $1 \mathrm{~min}$.) alone, and combination therapy of PDT with the topically administered angiogenesis inhibitors: sunitinib, sorafenib, erlotinib or bevacizumab. All agents were applied twice (immediately and $24 \mathrm{~h}$ post PDT) to a surface of $0.2 \mathrm{~cm}^{2}$. The vasculature is visualized by FITC-dextran fluorescence angiography $(25 \mathrm{mg} / \mathrm{kg}$, $20 \mathrm{kD}, \lambda_{\mathrm{ex}}=470 \mathrm{~nm}, \lambda_{\mathrm{em}}>520 \mathrm{~nm}$ ). (B) Schematic representation of the image processing quantification method. An original fluorescence angiography (left image) undergoes the skeletonization process as visualized on the block scheme (right). The skeletonized image is also shown as a map of distance of each pixel to the closest vascular segment, also referred to as 'potential oxygenation map'. Black areas represent avascular zones. The PDT area is indicated and divided into circular areas, 1 indicates the innermost area, 4 indicates the most peripheral area. (D) Schematic representation of the image processing procedure used to characterize the CAM vascular network. It indicates how image B was obtained from the information of image A. (C) Quantifications of three descriptors for two concentrations of the drugs. Means are shown, error bars represent standard error of the mean.

ranibizumab (Lucentis ${ }^{\circledR}$ ) or bevacizumab $\left(\right.$ Avastin $^{\circledR}$ ) after PDT can increase the duration of the closure of the vasculature [29]. We now demonstrate that similar results can be achieved with small molecule anti-angiogenic kinase inhibitors, which are currently used in the clinic.

As the CAM model is often used as a model for PDT-induced angio-occlusion, and the study of post-PDT revascularization, we developed an ImageJ-based vessel-quantification tool to digitally analyse vascular architecture and growth. As the revascularization of the CAM occurs from the edge of the PDT-treated area, we have now adopted the previously described software [25] to create four concentric circular areas. In each area the vascularization is monitored and quantified as a function of time after PDT, normalized per area. Regrowth of vessels starts from the existing vessels in the most outward circular area, and progresses into the other areas localized more towards the center of the PDT-treated area of the CAM surface. Various descriptors, such as vascular density and branching points per $\mathrm{mm}^{2}$ (Materials and methods), are used to quantify and characterize the revascularization in these areas. Results from this vessel quantification tool can be used to quantitatively test for optimal angio-occlusion and to optimize the combination of photodynamic angio-occlusion with angiogenesis 

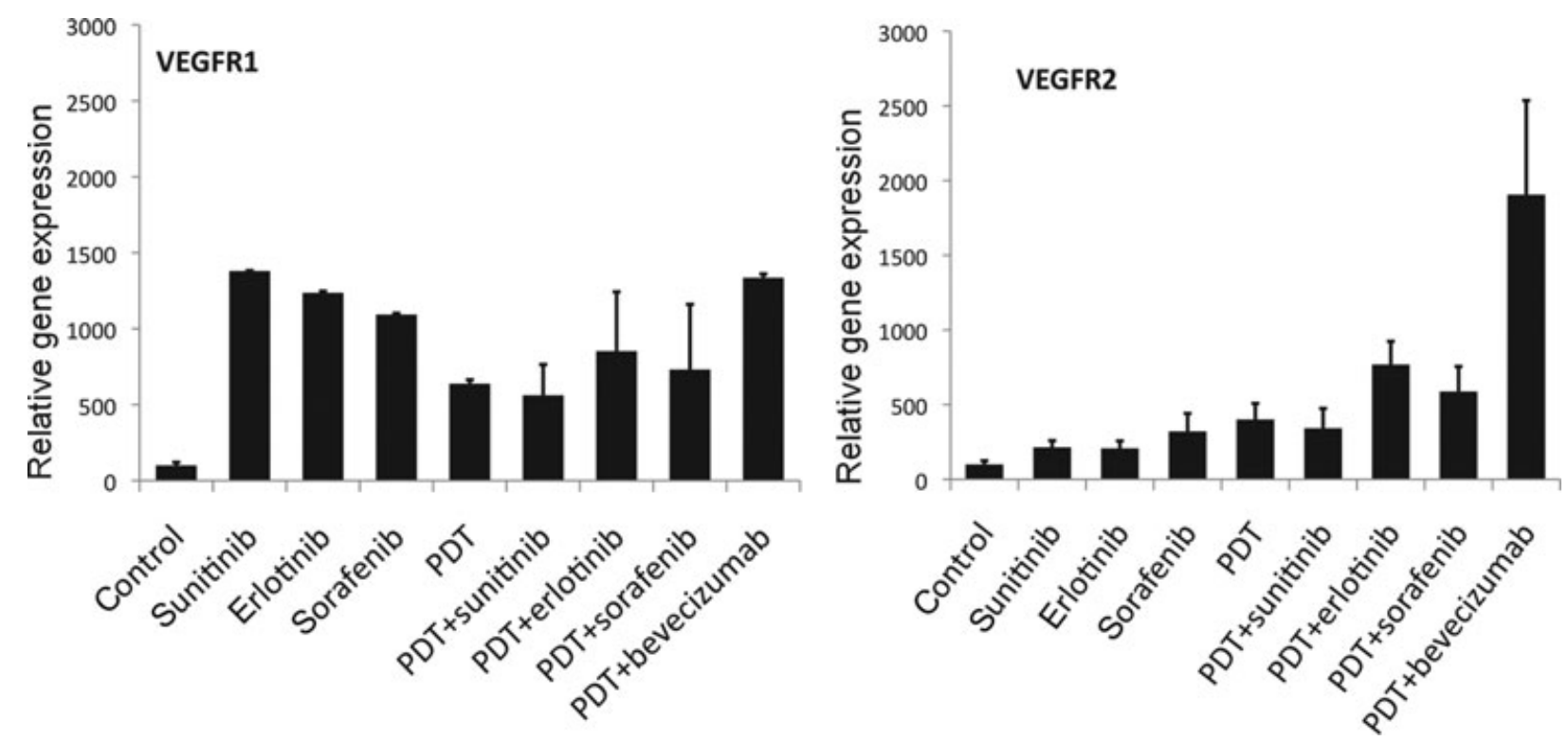

Fig. 4 Molecular regulation of VEGF receptors after PDT in combination with treatment with angiogenesis inhibitors. Quantitative real-time PCR using chicken-specific primers for the VEGFR1 and VEGFR2. Mean relative expressions are shown \pm standard error of the mean.

inhibitors. Following the angio-occlusive PDT, treatment with bevacizumab or kinase inhibitors resulted in significant inhibition of the post-PDT vascular regrowth. A change in density, as well as architectural differences of the vasculature after treatment was evident from all descriptors. The number of branching points in the vasculature (descriptor 1) was significantly repressed, whereas the parameter representing vascular mesh size was increased, suggesting a decrease in potential oxygenation, and the vessel density (descriptor 3). The results showed that the kinase inhibitors were very efficient inhibitors of vascular regrowth, and their activities were at least comparable to the activity of bevacizumab. Interestingly, sorafenib showed a significantly better activity as compared to treatment with bevacizumab, resulting in a significantly slower revascularization.

At the molecular level, it was observed that PDT treatment efficiently induced the expression of VEGFR1 and VEGFR2. Also the expression levels of VEGF and bFGF tended to go up, although not significantly. Co-treatment with angiogenesis inhibitors was found to further force the cells into an angiogenesis program [23]. These results demonstrate that both PDT and inhibitors of angiogenesis force the vasculature into a compensatory mechanism of angiogenesis stimulation.

For use in the treatment of eye diseases such as exudative AMD, there may be a number of advantages for the use of small molecule kinase inhibitors over bevacizumab. First of all, treatment with bevacizumab depends so far on intraocular injections, probably because of the low-tissue penetration of the antibody. As this is an invasive procedure, the use of a small molecule that may be administered through eye drops or sustained drug delivery systems [37-39] could have a significant benefit. It is anticipated that (i) systemic side-effects known for angiostatic agents will not occur because of the local, that is low dose, administration, (ii) that resistance to the drug will not evolve because the angiogenesis process is not tumour driven [40] and that (iii) vascular normalization is induced locally resulting in enhanced efficacy of the combination with PDT [41].

To support the assumption that the activity seen in the CAM is based on an activity on endothelial cells, and to be able to compare activities of the different anti-angiogenic compounds, we performed proliferation and migration assays making use of HUVEC. Although all kinase inhibitors inhibited proliferation with ED50's of $3-30 \mu \mathrm{M}$, sunitinib was the only kinase inhibitor that significantly inhibited migration with $\mathrm{ED}_{50}$ as low as $1-3 \mu \mathrm{M}$. It is interesting to observe that sorafenib, while inhibiting migration and proliferation only at a higher dose, seems to be the most effective inhibitor in both the developmental and the PDT CAM assays. A possible explanation for this phenomenon might be the in vitro versus in vivo difference, or the species difference in these assays, as sorafenib might have a higher affinity for chicken receptors as compared to their human counterparts. It is assumed that the activity of the compound is mainly on the endothelium, however, it cannot be excluded that the effect is indirect through an activity on epithelial cells, fibroblasts or vascular support cells. It should be realized that in this study we applied the small molecule kinase inhibitors topically on the CAM. This was done because to some extent this may be simulating application through eye drops. However, we know that i.v. injections of the kinase inhibitors works very efficiently as well, and seems to need even lower concentrations of the drug (NowakSliwinska et al., unpublished data). 


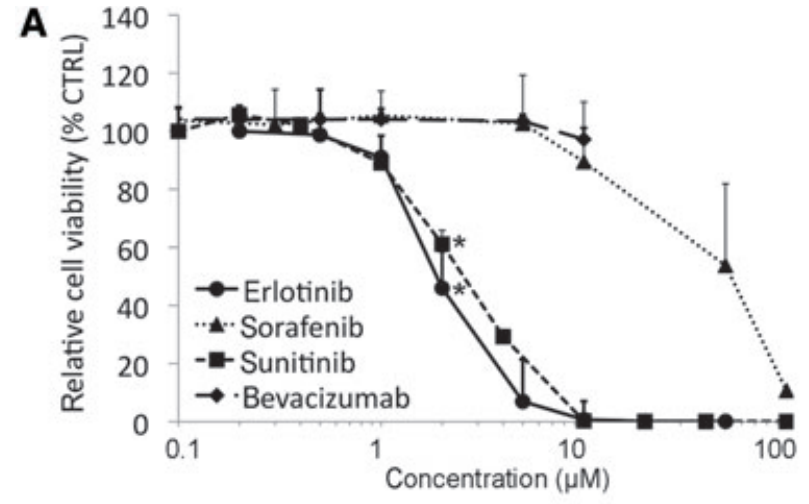

B

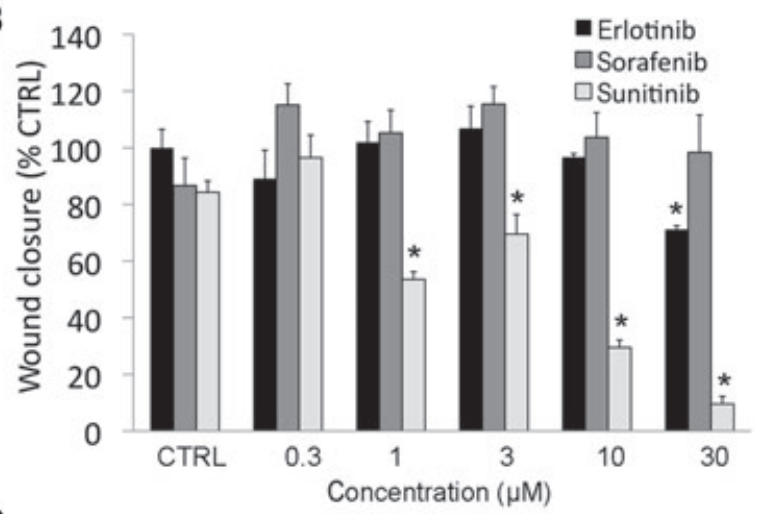

C

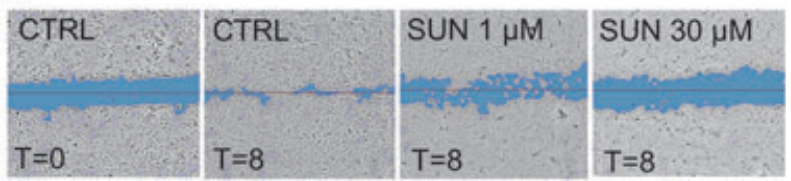

Fig. 5 Regulation of endothelial cell growth and migration. (A) Effect of the angiogenesis inhibitors on the proliferation of human umbilical vein endothelial cells (HUVEC). (B) Quantification of wound closure in HUVEC cultures after $8 \mathrm{hrs}$ of incubation with kinase inhibitors. Asterisks represent statistically significant differences as compared to the control $(P<0.05)$. (C) Typical images of the scratch wound at the beginning of the experiment and after $8 \mathrm{hrs}$ without and with two concentrations of sunitinib.

An alternative explanation for the good activity of sorafenib is the broader kinase spectrum, because VEGFR3 and B-Raf are kinase targets that are not shared by the other drugs. It remains to be seen if the benefit of sorafenib holds when it comes to application for eye diseases in patients.

Another interesting finding of this research is that the inhibitory dose of all angiogenesis inhibitors tested was approximately 10-fold lower in the PDT-induced angiogenesis, as compared to the developmental angiogenesis. We have previously shown a similar phenomenon for ruthenium-arene-based compounds possessing anti-angiogenic properties [27]. We hypothesize that this shows that developmental angiogenesis is a more robust process that is probably more intricately regulated, and more well-balanced, than the process that follows the sudden PDT challenge. It is furthermore tempting to speculate that whereas developmental angiogenesis is regulated by VEGF, angiopoietins, EGF, PDGF, PIGF, etc., PDT-induced angiogenesis is mainly driven by VEGF, as is the case in tumours. This would then result in a vasculature that has the tortuosity of tumour vasculature, an observation that we have published recently [23].

\section{Acknowledgements}

The authors are grateful for financial support from Dr. Julia Jacobi. This work was also supported by a grant from the Dutch Science Foundation (NWO 40.11.195) and the Swiss Federal Institute of Technology (EPFL).

\section{Conflict of interest}

The authors confirm that there are no conflicts of interest.

\section{Authors' contributions}

PNS and AWG designed the research study, performed the research, analysed data, and wrote the manuscript; AW and JRvB performed the research, analysed data and edited the manuscript, JPB and BL contributed to image processing method and analysed data, TJW performed the research, HvdB analysed data and edited the manuscript.

\section{References}

1. Folkman J. Angiogenesis in cancer, vascular, rheumatoid and other disease. Nat Med. 1995; 1: 27-31.

2. Griffioen AW, Molema G. Angiogenesis: potentials for pharmacologic intervention in the treatment of cancer, cardiovascular diseases, and chronic inflammation. Pharmacol Rev. 2000; 52: 237-68.

3. van den Bergh H. Photodynamic therapy of age-related macular degeneration: history and principles. Semin Ophthalmol. 2001; 16: 181-200.
4. Schlotzer-Schrehardt U, Viestenz A, Naumann GO, et al. Dose-related structural effects of photodynamic therapy on choroidal and retinal structures of human eyes. Graefes Arch Clin Exp Ophthalmol. 2002; 240: 748-57. 
5. Moshfeghi DM, Kaiser PK, Grossniklaus $\mathrm{HE}$, et al. Clinicopathologic study after submacular removal of choroidal neovascular membranes treated with verteporfin ocular photodynamic therapy. $A m \mathrm{~J}$ Ophthalmol. 2003; 135: 343-50.

6. Ju M, Mailhos C, Bradley J, et al. Simultaneous but not prior inhibition of VEGF165 enhances the efficacy of photodynamic therapy in multiple models of ocular neovascularization. Invest Ophthalmol Vis Sci. 2008; 49: 662-70.

7. Kaiser PK. Verteporfin photodynamic therapy and anti-angiogenic drugs: potential for combination therapy in exudative age-related macular degeneration. Curr Med Res Opin. 2007; 23: 477-87.

8. Cao Y. Tumour angiogenesis and molecular targets for therapy. Front Biosci. 2009; 14: 3962-73.

9. Hanahan D, Weinberg RA. Hallmarks of cancer: the next generation. Cell. 2011; 144: 646-74.

10. Merenmies J, Parada LF, Henkemeyer M. Receptor tyrosine kinase signaling in vascular development. Cell Growth Differ. 1997; 8: 3-10.

11. Hellberg C, Ostman A, Heldin CH. PDGF and vessel maturation. Recent Results Cancer Res. 2010; 180: 103-14.

12. Grimminger F, Schermuly RT, Ghofrani HA. Targeting non-malignant disorders with tyrosine kinase inhibitors. Nat Rev Drug Discov. 2010; 9: 956-70.

13. Martinelli G, Soverini S, lacobucci I, et al. Intermittent targeting as a tool to minimize toxicity of tyrosine kinase inhibitor therapy. Nat Clin Pract Oncol. 2009; 6: 68-9.

14. Weisberg E, Manley PW, Breitenstein W, et al. Characterization of AMN107, a selective inhibitor of native and mutant Bcr-Abl. Cancer Cell. 2005; 7: 129-41.

15. Motzer RJ, Hudes G, Wilding G, et al. Phase I trial of sunitinib malate plus interferon-alpha for patients with metastatic renal cell carcinoma. Clin Genitourin Cancer. 2009; 7: 28-33.

16. Faivre S, Raymond $\mathrm{E}$, Boucher $\mathrm{E}$, et al. Safety and efficacy of sunitinib in patients with advanced hepatocellular carcinoma: an open-label, multicentre, phase II study. Lancet Oncol. 2009; 10: 794-800.

17. Ferry DR, Anderson M, Beddard K, et al. A phase II study of gefitinib monotherapy in advanced esophageal adenocarcinoma: evidence of gene expression, cellular, and clinical response. Clin Cancer Res. 2007; 13: 5869-75.

18. Burger RA. Overview of anti-angiogenic agents in development for ovarian cancer. Gynecol Oncol. 2011; 121: 230-8.

19. Van den Eynde M, Baurain JF, Mazzeo F, et al. Epidermal growth factor receptor targeted therapies for solid tumours. Acta Clin Belg. 2011; 66: 10-7.

20. Iyer R, Fetterly G, Lugade A, et al. Sorafenib: a clinical and pharmacologic review. Expert Opin Pharmacother. 2010; 11: 1943-55.

21. Powles $\mathrm{T}$, Chowdhury $\mathbf{S}$, Jones $\mathbf{R}$, et al. Sunitinib and other targeted therapies for renal cell carcinoma. Br J Cancer. 2011; 104: 741-5.

22. Sulkes A. Novel multitargeted anticancer oral therapies: sunitinib and sorafenib as a paradigm. Israel Med Assoc J. 2010; 12: 628-32.

23. Nowak-Sliwinska $P$, van Beijnum J, van Berkel M, et al. Vascular regrowth following photodynamic therapy in the chicken embryo chorioallantoic membrane. Angiogenesis. 2010; 13: 281-92.

24. Lange N, Ballini JP, Wagnieres G, et al. A new drug-screening procedure for photosensitizing agents used in photodynamic therapy for CNV. Invest Ophthalmol Vis Sci. 2001; 42: 38-46.

25. Nowak-Sliwinska P, Ballini JP, Wagnieres $G$, et al. Processing of fluorescence angiograms for the quantification of vascular effects induced by anti-angiogenic agents in the CAM model. Microvasc Res. 2010; 79: 21-8.

26. Lim SH, Nowak-Sliwinska P, Kamarulzaman $F A$, et al. The neovessel occlusion efficacy of 15-hydroxypurpurin-7-lactone dimethyl ester induced with photodynamic therapy. Photochem Photobiol. 2010; 86: 397-402.

27. Nowak-Sliwinska $\mathbf{P}$, van Beijnum JR, Casini $A$, et al. Organometallic ruthenium(II)-arene compounds with antiangiogenic activity. J Med Chem. 2011; 54 : 3895-902.

28. Debefve E, Pegaz B, van den Bergh H, et al. Video monitoring of neovessel occlusion induced by photodynamic therapy with verteporfin (Visudyne), in the CAM model. Angiogenesis. 2008; 11: 235-43.

29. Debefve E, Pegaz B, Ballini JP, et al. Combination therapy using verteporfin and ranibizumab; optimizing the timing in the CAM model. Photochem Photobiol. 2009; 85: $1400-8$.

30. Pfaffl MW. A new mathematical model for relative quantification in real-time RT-PCR. Nucleic Acids Res. 2001; 29: e45.

31. van Beijnum JR, Dings RP, van der Linden $\mathrm{E}$, et al. Gene expression of tumour angiogenesis dissected: specific targeting of colon cancer angiogenic vasculature. Blood. 2006; 108: 2339-48.

32. Brandwijk RJ, Nesmelova I, Dings RP, et al. Cloning an artificial gene encoding angiostatic anginex: from designed peptide to functional recombinant protein. Biochem Biophys Res Commun. 2005; 333: $1261-8$.

33. van der Schaft DW, Dings RP, de Lussanet $\mathbf{Q G}$, et al. The designer antiangiogenic peptide anginex targets tumour endothelial cells and inhibits tumour growth in animal models. FASEB J. 2002; 16: 1991-3.

34. Lim SH, Thivierge C, Nowak-Sliwinska P, et al. In vitro and in vivo photocytotoxicity of boron dipyrromethene derivatives for photodynamic therapy. J Med Chem. 2010; 53: 2865-74.

35. Bressler NM. Antiangiogenic approaches to age-related macular degeneration today. Ophthalmology. 2009; 116: S15-23.

36. Ribatti D, Nico B, Vacca A, et al. Chorioallantoic membrane capillary bed: a useful target for studying angiogenesis and anti-angiogenesis in vivo. Anat Rec. 2001; 264: 317-24.

37. Yasukawa T, Ogura Y, Sakurai E, et al. Intraocular sustained drug delivery using implantable polymeric devices. Adv Drug Deliv Rev. 2005; 57: 2033-46.

38. Lee SS, Robinson MR. Novel drug delivery systems for retinal diseases: a review. Ophthalmic Res. 2009; 41: 124-35.

39. Nowak-Sliwinska P, Storto M, Cataudella $\mathrm{T}$, et al. Anti-angiogenic effects induced by the maleimide-based small molecule GNX686; 2011. Submitted.

40. Griffioen AW. Therapeutic approaches of angiogenesis inhibition: are we tackling the problem at the right level? Trends Cardiovasc Med. 2007; 17: 171-6.

41. Nowak-Sliwinska P, Wagnieres G, van den Bergh $\mathrm{H}$, et al. Angiostasis-induced vascular normalization can improve photodynamic therapy. Cell Mol Life Sci. 2010; 67: 1559-60. 